\title{
FURTHER GENERALIZATIONS OF THE NEHARI INEQUALITIES
}

\author{
BY
}

DUANE W. DeTEMPLE

\begin{abstract}
Inequalities of the Nehari type are obtained for bounded univalent functions on the unit disc, including a form which depends upon the parameters $a$ and $d$, where $d=f(a)$.
\end{abstract}

0. Introduction. The Nehari inequalities have been applied with considerable success to the study of coefficient problems for bounded univalent functions. It seems, however, that for further progress we must look to generalizations upon Nehari's original formulation; one need only look at the recent developments within the class of (unbounded) univalent functions which have come about by generalizing the Grunsky inequalities. Significant generalizations of the Nehari inequalities have, in fact, already appeared (Schiffer and Tammi [8], Nehari [7], DeTemple [1], [3]), and it is our purpose here to make a further contribution in this direction.

We obtain first some inequalities for the subclass of odd univalent functions which result from the square root transformation $f(z) \rightarrow \sqrt{ } f\left(z^{2}\right)$. These inequalities include a bilinear formulation which is employed in the second section to derive a set of inequalities which depend on two parameters $a$ and $d$, where $d=$ $f(a)$. More cumbersome inequalities of this type were demonstrated in DeTemple [3], where it was necessary to bring in the methods of the calculus of variations. By contrast, the results of the present paper are derived as corollaries of the Nehari inequalities.

1. Nehari inequalities for $\sqrt{ } f\left(z^{2}\right)$. Let $S_{1}$ denote the class of functions $f(z)=b_{1} z+b_{2} z^{2}+\cdots$ which are analytic and univalent mappings of the unit disc into itself. Thus $|f(z)|<1$ in $|z|<1$ and $b_{1}$ can be assumed to lie in the interval $0<$ $b_{1} \leqslant 1$. The following inequalities (Nehari [6]) are then necessary and sufficient for a given analytic function $f(z)$ to belong to $S_{1}$ :

$$
\left|\sum_{\mu, \nu=1}^{N} a_{\mu \nu} \lambda_{\mu} \lambda_{\nu}\right| \leqslant \sum_{\nu=1}^{N} \frac{1}{\nu}\left|\lambda_{\nu}\right|^{2}-\sum_{\mu, \nu=1}^{N} b_{\mu \nu} \lambda_{\mu} \bar{\lambda}_{\nu} \quad(N=1,2, \cdots)
$$

Received by the editors February 27, 1974.

AMS (MOS)subject classifications (1970). Primary 30A32, $30 \mathrm{~A} 34$.

Key words and phrases. Univalent functions, bounded univalent functions, Grunsky inequalities, Nehari inequalities. 
where $\lambda_{1}, \lambda_{2}, \cdots$ are arbitrary complex numbers and the Nehari coefficients $a_{\mu \nu}$ and $b_{\mu \nu}$ are defined by the generating functions

$$
\begin{gathered}
\varphi(z, \zeta) \equiv \log \frac{f(z)-f(\zeta)}{z-\zeta}=\sum_{\mu, \nu=0}^{\infty} a_{\mu \nu} z^{\mu} \xi^{\nu} \\
\psi(z, \bar{\zeta}) \equiv-\log [1-f(z) \overline{f(\zeta)}]=\sum_{\mu, \nu=1}^{\infty} b_{\mu \nu} z^{\mu} \bar{\zeta}^{\nu} .
\end{gathered}
$$

It should be observed that the properties of univalence and boundedness of a function $f$ can be transferred into statements of the regularity of the functions $\varphi$ and $\psi$ on the bicylinder $|z|<1 \times|\xi|<1$.

For any given function $f \in S_{1}$ we now introduce the odd function $f^{+}$, defined by $f^{+}(z)=\sqrt{ } f\left(z^{2}\right)$. Because $f^{+} \in S_{1}$, we can immediately infer the inequalities

$$
\left|\sum_{\mu, \nu=1}^{N} a_{\mu \nu}^{+} \lambda_{\mu} \lambda_{\nu}\right| \leqslant \sum_{\nu=1}^{N} \frac{1}{\nu}\left|\lambda_{\nu}\right|^{2}-\sum_{\mu, \nu=1}^{N} b_{\mu \nu}^{+} \lambda_{\mu} \bar{\lambda}_{\nu}
$$

where $a_{\mu \nu}^{+}$and $b_{\mu \nu}^{+}$are the Nehari coefficients for $f^{+}$. Curiously the inequalities (3) contain more than (1). Indeed, in light of the oddness of $f^{+}$, it is an easy matter to show

$$
\begin{array}{ll}
a_{2 \mu, 2 \nu}^{+}=1 / 2 a_{\mu \nu}, & a_{2 \mu+1,2 \nu}^{+}=a_{2 \mu, 2 \nu+1}^{+}=0, \\
b_{2 \mu, 2 \nu}^{+}=1 / 2 b_{\mu \nu}, & b_{2 \mu+1,2 \nu}^{+}=b_{2 \mu, 2 \nu+1}^{+}=0,
\end{array}
$$

which means (1) can be recovered from (3) by choosing $\lambda_{2 \nu+1}=0$. On the other hand, the coefficient combinations $a_{2 \mu+1,2 \nu+1}^{+}$and $b_{2 \mu+1,2 \nu+1}^{+}$are new, and we have at our disposal the additional inequalities

$$
\left|\sum_{\mu, \nu=0}^{N} a_{2 \mu+1,2 \nu+1}^{+} x_{\mu} x_{\nu}\right| \leqslant \sum_{\nu=0}^{N} \frac{1}{2 \nu+1}\left|x_{\nu}\right|^{2}-\sum_{\mu, \nu=0}^{N} b_{2 \mu+1,2 \nu+1}^{+} x_{\mu} \bar{x}_{\nu}
$$

where $x_{0}, x_{1}, \cdots$ are arbitrary complex numbers. For convenience we note that this inequality can also be recast in the matrix form

$$
\left|x^{t} A^{+} x\right| \leqslant x^{t} \sqrt{x}-x^{t} B^{+} \bar{x}
$$

where

$$
\begin{gathered}
A^{+}=\left(\left(a_{2 \mu+1,2 \nu+1}^{+}\right)\right)_{\mu, \nu=0}^{N}, \quad B^{+}=\left(\left(b_{2 \mu+1,2 \nu+1}^{+}\right)\right)_{\mu, \nu=0,}^{N}, \\
N=\left(\left(\frac{1}{2 \nu+1} \delta_{\mu \nu}\right)\right)_{\mu, \nu=0}^{N}, \quad x^{t}=\left(x_{0}, x_{1}, \cdots ; x_{N}\right) ;
\end{gathered}
$$

here $x^{t}$ is the transpose of the column vector $x$.

To obtain a bilinear form of (5), we replace $x$ by $x+h y, h$ real. In view 
of the symmetry of $A^{+}$and the hermitian symmetry of $B^{+}$we find

Thus

$$
\begin{aligned}
& \left|x^{t} A^{+} x+2 h x^{t} A^{+} y+h^{2} y^{t} A^{+} y\right| \\
& \quad \leqslant x^{t}\left(N-B^{+}\right) \bar{x}+2 h \operatorname{Re} x^{t}\left(N-B^{+}\right) \bar{y}+h^{2} y^{t}\left(N-B^{+}\right) \bar{y}
\end{aligned}
$$

$$
\begin{aligned}
4 h\left|x^{t} A^{+} y\right| & \leqslant\left|\left(x^{t} A^{+} x+2 h x^{t} A^{+} y+h^{2} y^{t} A^{+} y\right)-\left(x^{t} A^{+} x-2 h x^{t} A^{+} y+h^{2} y^{t} A^{+} y\right)\right| \\
& \leqslant 2 x^{t}\left(N-B^{+}\right) \bar{x}+2 h^{2} y^{t}\left(N-B^{+}\right) \bar{y}
\end{aligned}
$$

for all real $h$. The discriminant must then be nonpositive and so

$$
\left|x^{t} A^{+} y\right|^{2} \leqslant\left(x^{t}\left(N-B^{+}\right) \bar{x}\right)\left(y^{t}\left(N-B^{+}\right) \bar{y}\right) \text {. }
$$

An alternative way to obtain bilinear Nehari inequalities is to consider the form

$$
L(x, y)=\operatorname{Re}\left(x^{t} A^{+} y+x^{t} B^{+} y\right)
$$

for which (5) implies $|L(x)| \leqslant x^{t} N \bar{x}$; here $L(x) \equiv L(x, x)$. Moreover it easy to verify that

$$
\begin{aligned}
L(x, y) & =L(y, x), \\
L(\alpha x, \beta y) & =\alpha \beta L(x, y), \quad \alpha, \beta \text { real, } \\
L(x \pm y) & =L(x)+L(y) \pm 2 L(x, y), \\
L(x, y) & =1 / 4 L(x+y)-1 / 4 L(x-y),
\end{aligned}
$$

and so

$$
|L(x, y)| \leqslant 1 / 4(x+y)^{t} N(\bar{x}+\bar{y})+1 / 4(x-y)^{t} N(\bar{x}-\bar{y})=1 / 2 x^{t} N \bar{x}+1 / 2 y^{t} N \bar{y} .
$$

A stronger form is obtained upon replacing $x$ by $x /\left(x^{t} N \bar{x}\right)^{1 / 2}$ and $y$ by $y /\left(y^{t} N \bar{y}\right)^{1 / 2}$. The homogeneity of $L$ then gives $|L(x, y)|^{2} \leqslant\left(x^{t} N \bar{x}\right)\left(y^{t} N \bar{y}\right)$. Finally we replace $x$ by $e^{i(\varphi+\theta)} x$ and $y$ by $e^{i(\varphi-\theta)} y$, with $\varphi$ and $\theta$ real. Because

$$
L\left(e^{i(\varphi+\theta)} x, e^{i(\varphi-\theta)} y\right)=\operatorname{Re}\left(e^{2 i \varphi} x^{t} A^{+} y+e^{2 i \theta} x^{t} B^{+} y\right),
$$

the arbitrariness of $\varphi$ and $\theta$ allows us to conclude

In particular,

$$
\left|x^{t} A^{+} y\right|+\left|x^{t} B^{+} \bar{y}\right| \leqslant\left(x^{t} N \bar{x}\right)^{1 / 2}\left(y^{t} N \bar{y}\right)^{1 / 2} .
$$

$$
\operatorname{Re}\left(x^{t} A^{+} y+x^{t} B^{+} \bar{y}\right) \leqslant\left(x^{t} N \bar{x}\right)^{1 / 2}\left(y^{t} N \bar{y}\right)^{1 / 2}
$$

and so if we choose $x=N^{-1} \overline{\left(A^{+} y+B^{+} \bar{y}\right)}$ we find

(8) $\sum_{\mu=0}^{N}(2 \mu+1)\left|\sum_{\nu=0}^{N} a_{2 \mu+1,2 \nu+1}^{+} y_{\nu}+b_{2 \mu+1,2 \nu+1}^{+} \bar{y}_{\nu}\right|^{2} \leqslant \sum_{\nu=0}^{N} \frac{1}{2 \nu+1}\left|y_{\nu}\right|^{2}$.

We collect our results in the following theorem.

THEOREM 1. Let $f(z) \in S_{1}$ and define $f^{+}(z)=\sqrt{ } f\left(z^{2}\right)$. Define the elements of the matrices $A^{+}=\left(\left(a_{2 \mu+1,2 \nu+1}^{+}\right)\right)_{\mu, \nu=0}^{N}$ and $B^{+}=\left(\left(b_{2 \mu+1,2 \nu+1}^{+}\right)\right)_{\mu, \nu=0}^{N}$ by 
(9a) $\varphi^{+}(z, \zeta) \equiv \log \frac{\left[f^{+}(z)-f^{+}(\zeta)\right](z+\zeta)}{\left[f^{+}(z)+f^{+}(\zeta)\right](z-\zeta)}=2 \sum_{\mu, \nu=0}^{\infty} a_{2 \mu+1,2 \nu+1}^{+} z^{2 \mu+1} \zeta^{2 \nu+1}$,

(9b) $\psi^{+}(z, \bar{\zeta}) \equiv \log \frac{1+f^{+}(z) \overline{f^{+}(\zeta)}}{1-f^{+}(z) \overline{f^{+}(\zeta)}}=2 \sum_{\mu, \nu=0}^{\infty} b_{2 \mu+1,2 v+1}^{+} z^{2 \mu+1} \bar{\zeta}^{2 \nu+1}$.

Then, for arbitrary complex column vectors $x=\left(x_{\nu}\right)_{\nu=0}^{N}$ and $y=\left(y_{\nu}\right)_{\nu=0}^{N}$, the following equivalent inequalities hold, where $N=\left(\left(\delta_{\mu \nu} /(2 \nu+1)\right)\right)_{\mu, \nu=0}^{N}$ :

$$
\begin{gathered}
\left|x^{t} A^{+} x\right| \leqslant x^{t} N \bar{x}-x^{t} B^{+} \bar{x}, \\
\left|x^{t} A^{+} y\right|^{2} \leqslant\left(x^{t}\left(N-B^{+}\right) \bar{x}\right)\left(y^{t}\left(N-B^{+}\right) \bar{y}\right), \\
\left|x^{t} A^{+} y\right|+\left|x^{t} B^{+} \bar{y}\right| \leqslant\left(x^{t} N \bar{x}\right)^{1 / 2}\left(y^{t} N \bar{y}\right)^{1 / 2}, \\
\sum_{\mu=0}^{N}(2 \mu+1)\left|\sum_{\nu=0}^{N}\left(a_{2 \mu+1,2 \nu+1}^{+} x_{\nu}+b_{2 \mu+1,2 \nu+1}^{+} \bar{x}_{\nu}\right)\right|^{2} \leqslant x^{t} N \bar{x} .
\end{gathered}
$$

REMARKS. 1. An application of Schwarz's inequality shows that (10a) follows from (10d). The equivalence of the four inequalities is then apparent.

2. The inequalities continue to hold when $A^{+}, B^{+}, N$ and $x$ are replaced by $\left(\left(a_{\mu \nu}\right)\right)_{\mu, \nu=1}^{N},\left(\left(b_{\mu \nu}\right)\right)_{\mu, \nu=1}^{N},\left(\left(\delta_{\mu \nu} / v\right)\right)_{\mu, \nu=1}^{N},\left(x_{\nu}\right)_{\nu=1}^{N}$. Hummel [4] has also obtained results of this type.

3. For applications to coefficient problems it seems more convenient to replace (10b) and (10c) by the weaker forms

$$
\begin{gathered}
2 \operatorname{Re} x^{t} A^{+} y \leqslant x^{t}\left(N-B^{+}\right) \bar{x}+y^{t}\left(N-B^{+}\right) \bar{y}, \\
2 \operatorname{Re}\left(x^{t} A^{+} y+x^{t} B^{+} \bar{y}\right) \leqslant x^{t} N \bar{x}+y^{t} N \bar{y} .
\end{gathered}
$$

In the next section we shall see that $(10 \mathrm{~b})$ is required however.

2. Nehari inequalities for functions satisfying $d=f(a)$. The generating functions $\varphi^{+}$and $\psi^{+}$defined by (9) bear a close resemblance to some generating functions already introduced by DeTemple [1]. Indeed let

$$
\begin{gathered}
\Phi(z, \zeta) \equiv \log \frac{F(z)-F(\zeta)}{[F(z)+F(\zeta)](z-\zeta)}=\sum_{\mu, \nu=0}^{\infty} A_{\mu \nu} z^{\mu \zeta^{\nu}}, \\
\Psi(z, \bar{\zeta}) \equiv \log \frac{1+F(z) \overline{F(\zeta)}}{1-F(z) \overline{F(\zeta)}}=\sum_{\mu, \nu=0}^{\infty} B_{\mu \nu} z^{\mu \bar{\zeta}^{\nu}} .
\end{gathered}
$$

If $f \in S_{1}$ is a function which omits a value $d, 0<|d|<1$, then the above series converge in the bicylinder $|z|<1 \times|\xi|<1$ for the function

$$
F(z)=\sqrt{(d-f(z)) /(1-\bar{d} f(z))},
$$


and moreover we have the inequalities

$$
\operatorname{Re} \sum_{\mu, \nu=0}^{N}\left(A_{\mu \nu} \lambda_{\mu} \lambda_{\nu}+B_{\mu \nu} \lambda_{\mu} \bar{\lambda}_{\nu}\right) \leqslant \sum_{\nu=1}^{N} \frac{1}{\nu}\left|\lambda_{\nu}\right|^{2} \quad(N=1,2, \cdots) .
$$

Here $\lambda_{0}$ is any real number and the $\lambda_{1}, \lambda_{2}, \cdots$ are arbitrary complex numbers. Although the calculus of variations was used to prove (13) originally, a more elementary proof of area type was given in a subsequent paper [2].

Now suppose the parameter $d$ in (12) is taken as a value in the range of a function $f \in S_{1}$, so that $d=f(a)$ for some $a, 0<|a|<1$. For $|z|$ and $|\xi|$ sufficiently small, the series in (11) are still convergent. The left side of (13) can then still be formed, and for a fixed value $a$ we can seek its maximum. In fact this problem was solved in [3], but again by means of the calculus of variations.

We now show a more direct approach to such inequalities. The basic idea is one of Lebedev which arose in a similar context within the class $S$ of unbounded univalent functions (Lebedev [5]. For a given function $f \in S_{1}$ and a given point $a$ of the unit disc, $0<|a|<1$, define the odd bounded univalent function $f^{+}$by

$$
f^{+}(z)=\epsilon F\left(\left(a+z^{2}\right) /\left(1+\bar{a} z^{2}\right)\right),
$$

where $F$ is given by (12) with $d=f(a)$ and $\epsilon,|\epsilon|=1$, is a factor which insures $f^{+}(0)>0$.

Observing that $f^{+}(\sqrt{(z-a) /(1-\bar{a} z)})=\epsilon F(z)$, we have

$$
\begin{aligned}
& \varphi^{+}\left(\sqrt{\frac{z-a}{1-\bar{a} z}}, \sqrt{\frac{\zeta-a}{1-\bar{a} \zeta}}\right)=\log \frac{[F(z)-F(\zeta)]\left[\sqrt{\frac{z-a}{1-\bar{a} z}}+\sqrt{\frac{\zeta-a}{1-\bar{a} \zeta}}\right]}{[F(z)+F(\zeta)]\left[\sqrt{\frac{z-a}{1-\bar{a} z}}-\sqrt{\frac{\zeta-a}{1-\bar{a} \zeta}}\right]} \\
& \quad=\log \frac{F(z)-F(\zeta)}{[F(z)+F(\zeta)](z-\zeta)}-\log \frac{\sqrt{\frac{z-a}{1-\bar{a} z}}-\sqrt{\frac{\zeta-a}{1-\bar{a} \zeta}}}{\left[\sqrt{\frac{z-a}{1-\bar{a} z}}+\sqrt{\frac{\zeta-a}{1-\bar{a} \zeta}}\right](z-\zeta)}
\end{aligned}
$$

and similarly

$$
\psi^{+}\left(\sqrt{\frac{z-a}{1-\overline{a z}}}, \sqrt{\frac{\zeta-a}{1-\bar{a} \zeta}}\right)=\log \frac{1+F(z) \overline{F(\zeta)}}{1-F(z) \overline{F(\zeta)}} .
$$

As with Lebedev, we define the coefficients $\alpha_{\mu \nu}$ and $c_{\nu}^{(j)}$ by

$$
\log \frac{\sqrt{(1-z / a)(1-\bar{a} \zeta)}-\sqrt{(1-\zeta / a)(1-\bar{a} z)}}{[\sqrt{(1-z / a)(1-\bar{a} \zeta)}+\sqrt{(1-\zeta / a)(1-\bar{a} z)}](z-\zeta)}=\sum_{\mu, \nu=0}^{\infty} \alpha_{\mu \nu} z^{\mu} \zeta^{\nu}
$$

and 


$$
\left(\sqrt{\frac{z-a}{1-\overline{a z}}}\right)^{j}=\sum_{\mu=0}^{\infty} c_{\mu}^{(j) z^{\mu}} .
$$

The series expansions of equations (14), which are valid for sufficiently small $|z|$ and $|\zeta|$, can be expressed in terms of the coefficients introduced by equations (9), (11), (15) and (16). Comparing these expansions we see

$$
\begin{aligned}
A_{\mu \nu}-\alpha_{\mu \nu} & =2 \sum_{j, k=0}^{\infty} c_{\mu}^{(2 j+1)} c_{\nu}^{(2 k+1)} a_{2 j+1,2 k+1}^{+}, \\
B_{\mu \nu} & =2 \sum_{j, k=0}^{\infty} c_{\mu}^{(2 j+1)} \overline{c_{\nu}^{(2 k+1)}} b_{2 j+1,2 k+1}^{+}
\end{aligned}
$$

Then if $x_{0}, y_{0}, x_{1}, y_{1}, \cdots$ are arbitrary complex numbers, and $N$ is any nonnegative integer, we find

$$
\begin{aligned}
& \left|\sum_{\mu, \nu=0}^{N}\left(A_{\mu \nu}-\alpha_{\mu \nu}\right) x_{\mu} y_{\nu}\right|^{2} \\
& =\left|\sum_{\mu, \nu=0}^{N}\left(2 \sum_{j, k=0}^{\infty} c_{\mu}^{(2 j+1)} c_{\nu}^{(2 k+1)} a_{2 j+1,2 k+1}^{+}\right) x_{\mu} y_{\nu}\right|^{2} \\
& =\left|\sum_{j, k=0}^{\infty} a_{2 j+1,2 k+1}^{+}\left(\sum_{\mu=0}^{N} \sqrt{2} c_{\mu}^{(2 j+1)} x_{\mu}\right)\left(\sum_{\nu=0}^{N} \sqrt{2} c_{\nu}^{(2 k+1)} y_{\nu}\right)\right|^{2} \\
& \leqslant\left[\sum_{j=0}^{\infty} \frac{2}{2 j+1}\left|\sum_{\mu=0}^{N} c_{\mu}^{(2 j+1)} x_{\mu}\right|^{2}\right. \\
& \left.-2 \sum_{j, k=0}^{\infty} b_{2 j+1,2 k+1}^{+} \sum_{\mu=0}^{N} c_{\mu}^{(2 j+1)} x_{\mu} \overline{\sum_{\nu=0}^{N} c_{\nu}^{(2 k+1)} x_{\nu}}\right] \\
& \cdot\left[\sum_{j=0}^{\infty} \frac{2}{2 j+1}\left|\sum_{\mu=0}^{N} c_{\mu}^{(2 j+1)} y_{\mu}\right|^{2}\right. \\
& \left.-2 \sum_{j, k=0}^{\infty} b_{2 j+1,2 k+1}^{+} \sum_{\mu=0}^{N} c_{\mu}^{(2 j+1)} y_{\mu} \overline{\sum_{\nu=0}^{N} c_{\nu}^{(2 k+1)} y_{\nu}}\right] \text {, }
\end{aligned}
$$

where the inequality step above is a consequence of (10b).

The final form of our inequalities will be reached by simplifying the righthand side of (17). We first notice that 


$$
\begin{aligned}
2 \sum_{j, k=0}^{\infty} b_{2 j+1,2 k+1}^{+} & \sum_{\mu=0}^{N} c_{\mu}^{(2 j+1)} x_{\mu} \overline{\sum_{\nu=0}^{N} c_{\nu}^{(2 k+1)} x_{\nu}} \\
& =\sum_{\mu, \nu=0}^{N}\left(2 \sum_{j, k=0}^{\infty} c_{\mu}^{(2 j+1) \overline{c_{\nu}^{(2 k+1)}}} b_{2 j+1,2 k+1}^{+}\right) x_{\mu} \bar{x}_{\nu} \\
& =\sum_{\mu, \nu=0}^{N} B_{\mu \nu} x_{\mu} \bar{x}_{\nu} .
\end{aligned}
$$

Next we define the coefficients $\beta_{\mu \nu}$ by

$$
\log \frac{\sqrt{(1-\bar{a} z)(1-a \bar{\zeta})}+|a| \sqrt{(1-z / a)(1-\bar{\zeta} / a)}}{\sqrt{(1-\bar{a} z)(1-a \bar{\zeta})}-|a| \sqrt{(1-z / a)(1-\bar{\zeta} / a)}}=\sum_{\mu, \nu=0}^{\infty} \beta_{\mu \nu} z^{\mu} \bar{\zeta}^{\nu}
$$

which allows us to write

$$
\begin{aligned}
& \sum_{j=0}^{\infty} \frac{2}{2 j+1}\left|\sum_{\mu=0}^{N} c_{\mu}^{(2 j+1)} x_{\mu}\right|^{2} \\
& \quad=\sum_{\mu, \nu=0}^{N}\left(\sum_{j=0}^{\infty} \frac{2}{2 j+1} c_{\mu}^{(2 j+1)} \overline{c_{\nu}^{(2 j+1)}}\right) x_{\mu} \bar{x}_{\nu} \\
& \quad=\sum_{\mu, \nu=0}^{N} \beta_{\mu \nu} x_{\mu} \bar{x}_{\nu} .
\end{aligned}
$$

Making these substitutions into inequality (17) yields the following result.

THEOREM 2. Let $f \in S_{1}, 0<|a|<1$, and $d=f(a)$. If the coefficients $A_{\mu \nu}, B_{\mu \nu}, \alpha_{\mu \nu}, \beta_{\mu \nu}$ are defined by equations (11), (12), (15) and (18), then the inequalities

$$
\left|\sum_{\mu, \nu=0}^{N}\left(A_{\mu \nu}-\alpha_{\mu \nu}\right) x_{\mu} y_{\nu}\right|^{2}
$$

$$
\leqslant\left[\sum_{\mu, \nu=0}^{N}\left(\beta_{\mu \nu}-B_{\mu \nu}\right) x_{\mu} \bar{x}_{\nu}\right]\left[\sum_{\mu, \nu=0}^{N}\left(\beta_{\mu \nu}-B_{\mu \nu}\right) y_{\mu} \bar{y}_{\nu}\right] \quad(N=0,1, \cdots)
$$

hold for any sequence of complex numbers $x_{0}, y_{0}, x_{1}, y_{1}, \cdots$.

REMARKs. 1. The inequality can be restated in various weaker forms, including for example

$$
\operatorname{Re} \sum_{\mu, \nu=0}^{N}\left(A_{\mu \nu} x_{\mu} x_{\nu}+B_{\mu \nu} x_{\mu} \bar{x}_{\nu}\right) \leqslant \operatorname{Re} \sum_{\mu, \nu=0}^{N}\left(\alpha_{\mu \nu} x_{\mu} x_{\nu}+\beta_{\mu \nu} x_{\mu} \bar{x}_{\nu}\right) .
$$

Here the parameter $d$ appears only in the left-hand side, and $a$ only on the right. 
2. The coefficients $\alpha_{\mu \nu}$ and $\beta_{\mu \nu}$, which depend only on $a=r e^{i \theta}$, can be expressed by means of the polynomials $P_{n}$ and $\Pi_{n}$ defined by

$$
\begin{aligned}
\left(1-2 \xi z+z^{2}\right)^{-1 / 2} & =\sum_{n=0}^{\infty} P_{n}(\xi) z^{n}, \\
\left(1-2 \xi z+z^{2}\right)^{1 / 2} & =\sum_{n=0}^{\infty} \Pi_{n}(\xi) z^{n} .
\end{aligned}
$$

Indeed, as shown by Lebedev [5], if $\xi=1 / 2(r+1 / r)$, then

$$
\begin{aligned}
& \mu \alpha_{\mu \nu}=-\sum_{n=\nu+1}^{\mu+\nu} P_{\mu+\nu-n}(\xi) \Pi_{n}(\xi) e^{-i(\mu+\nu) \theta}, \\
& \mu \beta_{\mu \nu}=-\sum_{n=0}^{\min \{\mu-1, \nu\}} P_{\mu-1-n}(\xi) \Pi_{n-\nu}(\xi) e^{i(\nu-\mu) \theta} .
\end{aligned}
$$

Of course $P_{n}(\xi)$ are the Legendre polynomials, while the $\Pi_{n}(\xi)$ are closely related to them. It is easily shown that $n \Pi_{n}(\xi)=P_{n-2}(\xi)-\xi P_{n-1}(\xi)$.

\section{REFERENCES}

1. D. W. DeTemple, On coefficient inequalities for bounded univalent functions, Ann. Acad. Sci. Fenn. Ser. A I No. 469 (1970). MR 43 \#5020.

2. - Grunsky-Nehari inequalities for a subclass of bounded univalent functions, Trans. Amer. Math. Soc. 159 (1971), 317-328. MR 43 \#5022.

3. - Generalizations of the Grunsky-Nehari inequalities, Arch. Rational Mech. Anal. 44 (1971), 93-120.

4. J. A. Hummel, Inequalities of Grunsky type for Aharonov pairs, J. Analyse Math. 25 (1972), 217-257. MR 47 \#455.

5. N. A. Lebedev, Certain corollaries of an inequality of Grunsky, Vestnik Leningrad Univ. No. 7 (1972), 45-55. (Russian) MR 45 \#8820.

6. Z. Nehari, Some inequalities in the theory of functions, Trans. Amer. Math. Soc. 75 (1953), 256-286. MR 15, 115.

7. Inequalities for the coefficients of univalent functions, Arch. Rational Mech. Anal. 34 (1969), 301-330. MR 40 \#330.

8. M. Schiffer and $O$. Tammi, On the coefficient problem for bounded univalent functions, Trans. Amer. Math. Soc. 140 (1969), 461-474. MR 39 \#7088.

DEPARTMENT OF MATHEMATICS, WASHINGTON STATE UNIVERSITY, PULLMAN, WASHINGTON 99163 